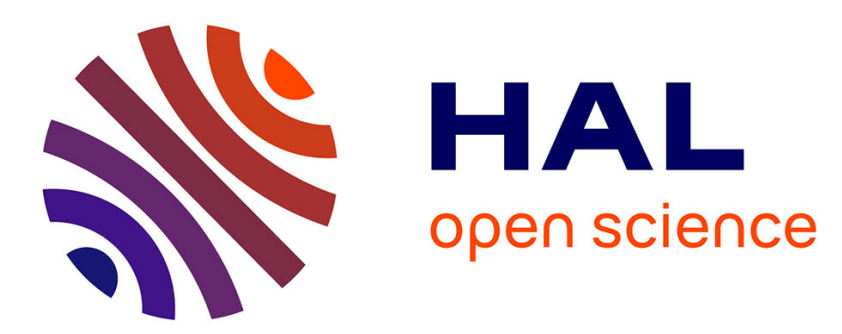

\title{
Relationship between Draw Ratio and Strain-Induced Crystallinity in Uniaxially Hot-Drawn PET MXD6 Films
}

\author{
Bessem Ben Doudou, Eric Dargent, Jean Grenet
}

\section{To cite this version:}

Bessem Ben Doudou, Eric Dargent, Jean Grenet. Relationship between Draw Ratio and StrainInduced Crystallinity in Uniaxially Hot-Drawn PET MXD6 Films. Journal of Plastic Film and Sheeting, 2005, 21 (3), pp.233-251. 10.1177/8756087905058248 . hal-00572064

\section{HAL Id: hal-00572064 \\ https://hal.science/hal-00572064}

Submitted on 1 Mar 2011

HAL is a multi-disciplinary open access archive for the deposit and dissemination of scientific research documents, whether they are published or not. The documents may come from teaching and research institutions in France or abroad, or from public or private research centers.
L'archive ouverte pluridisciplinaire HAL, est destinée au dépôt et à la diffusion de documents scientifiques de niveau recherche, publiés ou non, émanant des établissements d'enseignement et de recherche français ou étrangers, des laboratoires publics ou privés. 


\title{
ReLationship Between Draw Ratio and Strain-Induced CRYstallinity IN UNIAXIALLY HoT-DRAWN PET-MXD6 FILMS*
}

\section{Bessem Ben Doudou, Eric Dargent ${ }^{\dagger}$ and Jean Grenet}

Laboratoire PBM, UMR 6522, Equipe LECAP

Université de Rouen, Institut des Matériaux

Avenue de l'Université, B.P. 12

76801 Saint Etienne du Rouvray Cedex, France

\begin{abstract}
The evolutions of the strain-induced crystalline phase of poly(ethylene terephthalate) (PET), poly( $m$-xylene adipamide) (MXD6), and PET-MXD6-ionomer blend films are investigated by differential scanning calorimetry, X-ray diffraction, and birefringence measurements. Initially wholly amorphous, the films are uniaxially hot-drawn above the glass transition temperature $T_{\mathrm{g}}$. During drawing and depending on the draw ratio, an induced crystalline phase appears in the three materials. The comparison of the degree of crystallinity shows that the maximum degree of crystallinity is close to $40 \%$ for the three materials and the DSC analysis shows a similar thermal behavior between PET, MXD6, and blend samples. The critical draw ratios for the crystalline phase appearance are equal to 2, 3, and 3 for PET, MXD6, and blend, respectively.
\end{abstract}

KEY WORDS: PET, MXD6, blends, drawing, strain-induced crystallization.

\section{INTRODUCTION}

D OlYMer BLENDING IS a convenient method for the development of new polymeric materials, which combine the excellent properties of more than one existing polymer. This strategy is usually cheaper and

\footnotetext{
*This article was originally presented at the Films and Fibers 2004 Symposium of the National Research Council of Canada, which was held in Boucherville, Quebec, Canada in September 2004.

†Author to whom correspondence should be addressed. E-mail: eric.dargent@univ-rouen.fr
} 
Table 1. $\mathrm{O}_{2}$ and $\mathrm{CO}_{2}$ permeability for MXD6 films.

\begin{tabular}{lcc}
\hline & $\begin{array}{c}\text { Oxygen transmission rate }\left(\mathbf{2 3}^{\circ} \mathbf{C}\right) \\
\left(\mathbf{c m}^{3} / \mathbf{m}^{2} \text { day bars }\right) 25 \mu \mathbf{m}\end{array}$ & $\begin{array}{c}\text { Carbon dioxide transmission rate }(\mathbf{2 3} \mathbf{C}) \\
\left(\mathbf{c m}^{3} / \mathbf{m}^{2} \text { day bars }\right) \mathbf{2 5} \mu \mathbf{m}\end{array}$ \\
\hline PET & $45-90$ & $100-200$ \\
MXD6 & $1-2$ & $4-8$ \\
\hline
\end{tabular}

less time-consuming than the development of new polymers. In recent years, an increasing use of polyethylene terephtalate (PET) blends with barrier polymers (EVOH, PEN, PAs, etc.) have been taking place in the food industry to reduce the gas permeation through PET bottles and films [1]. Indeed, polymers with high barrier properties are required for packaging of carbonated soft drinks, where the permeation of carbon dioxide, oxygen, and water should be suppressed. In this work, we will study blends of PET and poly( $m$-xylene adipamide) (MXD6). This semiaromatic polyamide was chosen because it exhibits very high barrier properties for $\mathrm{CO}_{2}$ and $\mathrm{O}_{2}$ (see Table 1) and it has a melting temperature close to that of PET [2,3]. Owing to the immiscibility of these two classes of polymers, polyesters, and polyamides [4-6], it is necessary to compatibilize PET and MXD6 to obtain blends with good properties. In this work, the first attempt at blending was without a compatibilizer; all subsequent blends were made with an acrylic-modified polyolefin type ionomer (ion, $\mathrm{Zn}^{2+}$ ).

It is known that the barrier properties of polymers are controlled in part by their crystalline structure and their degree of crystallinity [7] because the crystalline phase is generally impermeable to small molecules. For this reason, in the present work, we have focused on the strain-induced crystallization (SIC) of the blends. For PET, this had been the subject of several works [8-15]. Concerning MXD6, very few studies were carried out on this polymer and a good knowledge of how it draws is missing. This is another aim of this study.

\section{EXPERIMENTAL}

The commercial grades of resins used in this study are listed in Table 2.

In this work, the compatibilization of PET with MXD6 is examined using an ionomer (ion, $\mathrm{Zn}^{2+}$ ), as the compatibilizer. This ionomer, $\mathrm{Zn}^{2+}$ (Surlyn 9020), is a random ethylene-methacrylic acid-isobutylacrylate terpolymer with the molar concentration of 78:10:12, respectively. The methacrylic acid is partially neutralized with zinc to $\approx 70 \%$. 
Table 2. Commercial grades used with density and measure of molecular weight.

\begin{tabular}{lclcl}
\hline Resin & Grade & \multicolumn{1}{c}{ Company } & $\begin{array}{c}\text { Density } \\
\mathbf{( g / \mathbf { c m } ^ { 3 } )}\end{array}$ & Molecular weight \\
\hline $\begin{array}{l}\text { Poly(ethylene } \\
\text { terephthalate) }\end{array}$ & PET T74F9 & Tergal Fibers & 1.4 & $\mathrm{IV}=0.71 \mathrm{dL} / \mathrm{g}$, \\
$\begin{array}{l}\text { Polyamide } \\
\text { MXD6 6007 }\end{array}$ & $\begin{array}{c}\text { Mitsubishi Gas } \\
\text { Chemical Co. }\end{array}$ & 1.22 & $M_{\mathrm{n}}=25,000 \mathrm{~g} \mathrm{~mol}^{-1}$ \\
lonomer, $\mathrm{Zn}^{2+}$ & Surlyn 9020 & $\begin{array}{c}\text { Du Pont de Nemours Co. } \\
0.96\end{array}$ & $M_{\mathrm{n}}=25,000 \mathrm{~g} \mathrm{~mol}^{-1}$ \\
\hline
\end{tabular}

The choice of this ionomer was dictated by the possibility for transreactions with PET $[16,17]$ and by its strong interactions with the amide groups of PA attributed to metal-ion coordination and specific forces, such as ion-dipole and H-bonding interactions $[18,19]$.

To avoid hydrolysis, PET pellets were dried in a vacuum oven at $130^{\circ} \mathrm{C}$ for $36 \mathrm{~h}$. Both MXD6 and zinc ionomer are dried at $70^{\circ} \mathrm{C}$ for $24 \mathrm{~h}$. Then, the dried PET pellets were dry-mixed with MXD6 and with or without compatibilizer pellets in the weight ratios: 88/10/2 (or 90/10/0). The mixture was extruded in a single-screw extruder. The temperature profile, starting from the feeding zone to the die, was $270,280,270$, and $250^{\circ} \mathrm{C}$, respectively, and the screw rotating rate was maintained at $30 \mathrm{rpm}$. The extrudate $(2 \mathrm{~mm}$ in diameter) was water cooled down to ambient temperature. The extruded blends of PET and MXD6 were then pelletized for injection molding experiments (pellet length of about $3 \mathrm{~mm}$ ). Injection molded plates $\left(4 \times 4 \times 0.2 \mathrm{~cm}^{3}\right)$ were prepared with melting and molding temperatures, respectively, of 270 and $40^{\circ} \mathrm{C}$ with the help of a lab-scale injection molding machine, 'Babyplast' (produced by Cronoplast S.L.). This procedure resulted in transparent plates.

Before the drawing period, the plates were placed for $15 \mathrm{~min}$ in the heating chamber of a tensile machine at $100^{\circ} \mathrm{C}$ to allow a homogeneous temperature distribution. This drawing temperature was chosen above the glass transition temperature $\left(T_{\mathrm{g}}\right)\left(75\right.$ and $85^{\circ} \mathrm{C}$ respectively for PET and MXD6, respectively) to allow a homogeneous drawing. The amorphous plates were uniaxially drawn with a drawing speed of $500 \mathrm{~mm} / \mathrm{min}$ using a tensile machine (Instron 4301). To freeze the orientation due to drawing, the sample was cold air quenched to room temperature. The draw ratio $\lambda$ of the drawn plates defined as the ratio of the extended length to the original length determined from displacement of ink marks, varied from 1 to 5.6 for the MXD6, from 1 to 6.6 for the PET, and from 1 to 4.8 for the blend. Morphologies of the cryogenically fractured surfaces were examined by scanning electron 
microscopy (SEM) using LEO 1530 XB. The MXD6 phase was etched out by formic acid followed by coating with gold prior to the SEM examination. The thermal behavior of the samples was examined with a Perkin-Elmer DSC7 calorimeter in a dry nitrogen atmosphere at the heating rate of $10^{\circ} \mathrm{C} / \mathrm{min}$. Temperature and energy calibrations of the conventional differential scanning calorimetry (DSC) were achieved using indium as a standard. The optical anisotropy of the samples was studied by birefringence measurements at room temperature, using a spectro photometric method [20]. Wide angle X-ray diffraction (XRD) measurements in reflection were carried out at room temperature with a Kigaru miniflex diffractometer system; a $\mathrm{Cu}$ anode was used as the $\mathrm{X}$ ray source $(\lambda=0.154 \mathrm{~nm})$. Data were collected in the range $2 \theta$ of $10-50^{\circ}$ using $0.02^{\circ}$ step and a counting time of $5 \mathrm{~s}$. In an unpublished work, we microtomed the sample parallel to the surfaces and showed that XRD patterns are the same in the surface and in the core. Hence, in this work we believe that the XRD patterns are representative of the whole samples and of the real crystalline morphology and structure.

\section{RESULTS AND DISCUSSION}

Figure 1 shows the DSC behavior of undrawn $(\lambda=1)$ PET, MXD6, and PET-MXD6 blend samples. The PET curve is the standard DSC curve expected for an amorphous PET material.

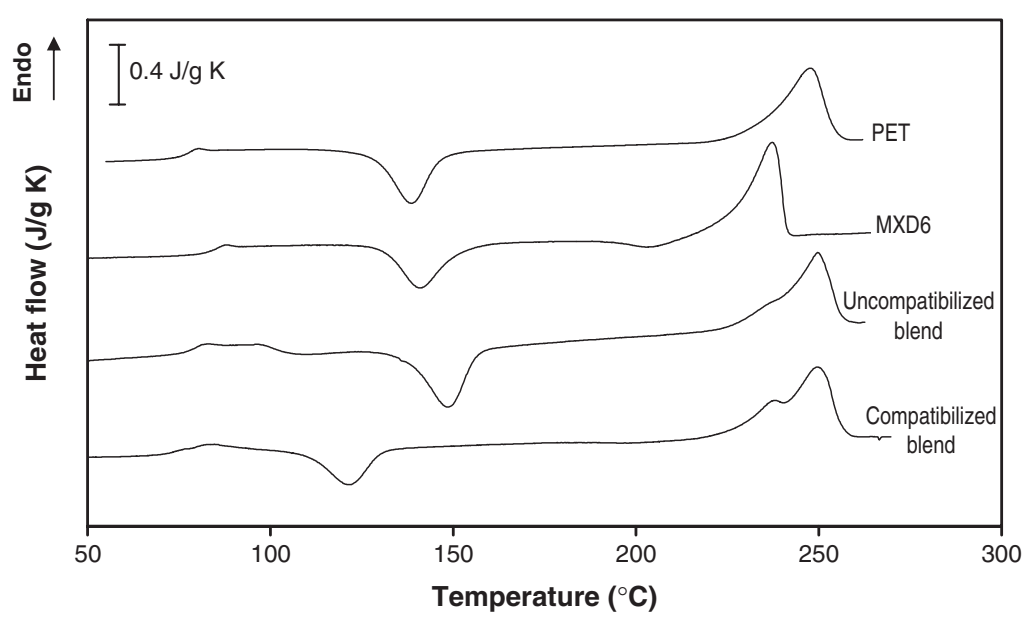

Figure 1. DSC curves for amorphous PET, MXD6, and blends (PET-MXD6 and PET-MXD6-ionomer). 
We can see the glass transition at $66^{\circ} \mathrm{C}<T_{\mathrm{g}}<86^{\circ} \mathrm{C}$, evidenced by the endothermic step, the thermal cold crystallization which is revealed by the exothermic peak at $114^{\circ} \mathrm{C}<T_{\mathrm{c}}<156^{\circ} \mathrm{C}$, and the endothermic peak occurring during the crystalline phase melting between 220 and $260^{\circ} \mathrm{C}$.

For MXD6, the curve shows the glass transition at $78^{\circ} \mathrm{C}<T_{\mathrm{g}}<93^{\circ} \mathrm{C}$. The thermal cold crystallization peak lies between 125 and $160^{\circ} \mathrm{C}$. Then a second exothermic phenomenon, generally attributed to a crystalline reorganization [21], appears around $T=205^{\circ} \mathrm{C}$. Finally, the melting of the crystalline phase occurs between 210 and $244^{\circ} \mathrm{C}$. It is noticed that MXD6 has practically the same melting temperature as PET $\left(T_{\max }=238^{\circ} \mathrm{C}\right.$ for $\mathrm{MXD} 6$ and $249^{\circ} \mathrm{C}$ for PET).

For uncompatibilized and compatibilized blends, the analysis shows a glass transition larger than the transition of pure materials $66^{\circ} \mathrm{C}<T<91^{\circ} \mathrm{C}$. The temperature difference between MXD6 and PET glass transition is too weak to be observed as two distinguished phenomena. The thermal cold crystallization peak appears at $132^{\circ} \mathrm{C}<$ $T_{\mathrm{c}}<162^{\circ} \mathrm{C}$ for the uncompatibilized blend. For the compatibilized blend, the thermal cold crystallization peak appears at a lower temperature $105^{\circ} \mathrm{C}<T_{\mathrm{c}}<140^{\circ} \mathrm{C}$ due to an interface effect. Finally, two melting peaks occur at 238 and $250^{\circ} \mathrm{C}$, corresponding to the fusion of the crystalline phases of the two components of the blend.

The morphology of the two components of the blend are shown in Figures 2 and 3. For the blend without compatibilizer, the holes representative of the MXD6 take the form of spherical inclusions with sizes ranged between 2 and $16 \mu \mathrm{m}$. For the compatibilized blend, the holes representative of MXD6 phase are more homogeneously

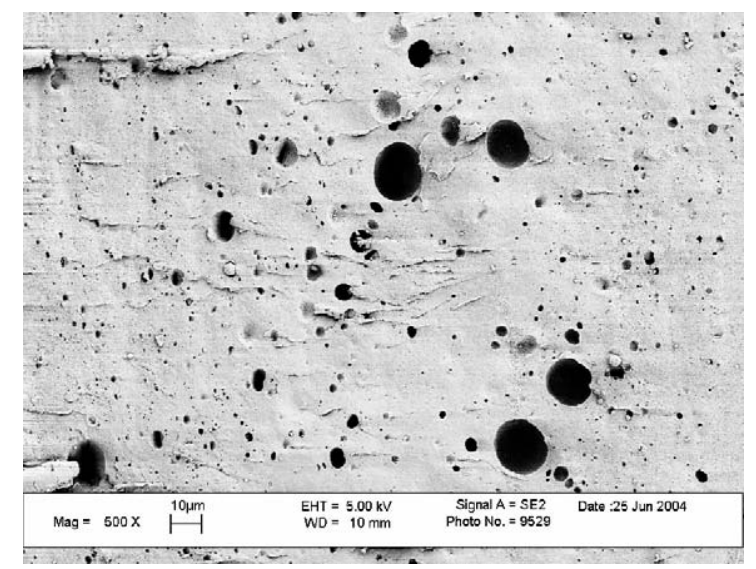

Figure 2. Scanning electron micrograph of cryofractured PET-MXD6 blends (90/10). 


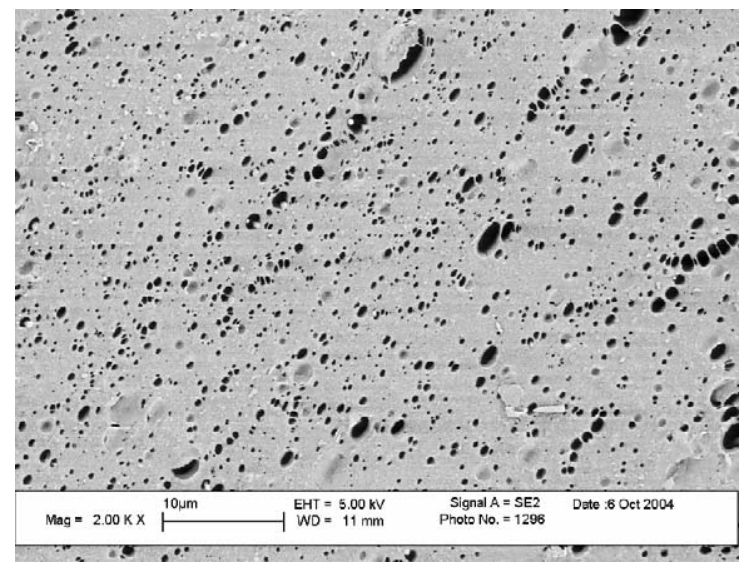

Figure 3. Scanning electron micrograph of cryofractured PET-MXD6-ion, $\mathrm{Zn}^{2+}$ blends $(88 / 10 / 2)$.

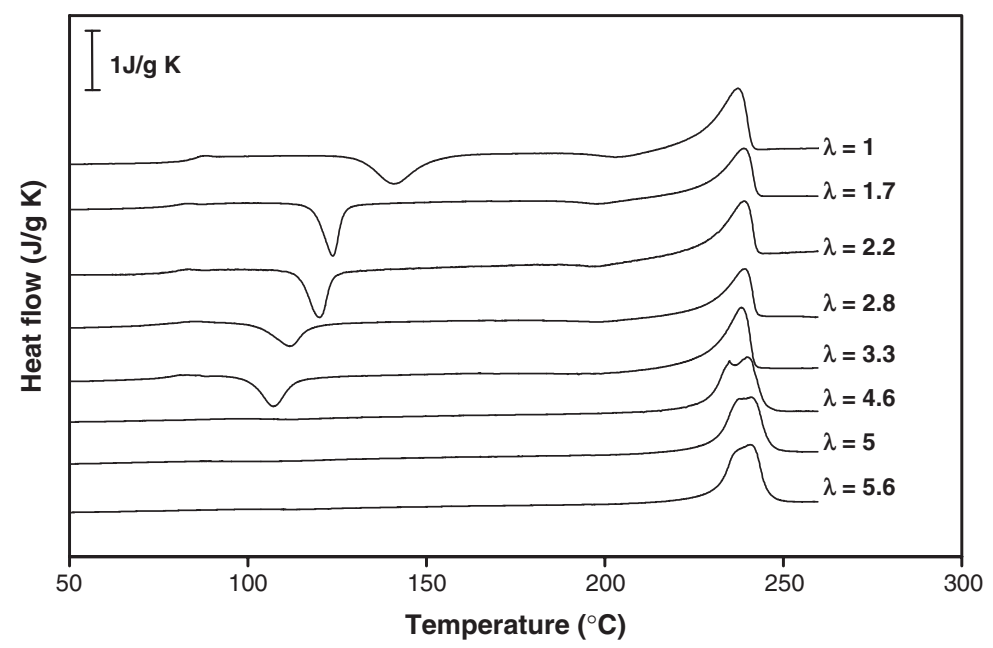

Figure 4. DSC normalized curves for MXD6 drawn at various draw ratios.

distributed in the PET matrix and they take the form of spherical inclusions. The sizes of the spheres ranged between 0.5 and $1.2 \mu \mathrm{m}$. After making this observation, we decided to study only blends that were compatibilized with the ionomer.

Previous articles [10-12] dealt with the influence of drawing on PET's DSC behavior, hence, in this article we focused our attention on the DSC curves of MXD6 (Figure 4) and the PET-MXD6-ionomer blend (Figure 5). 


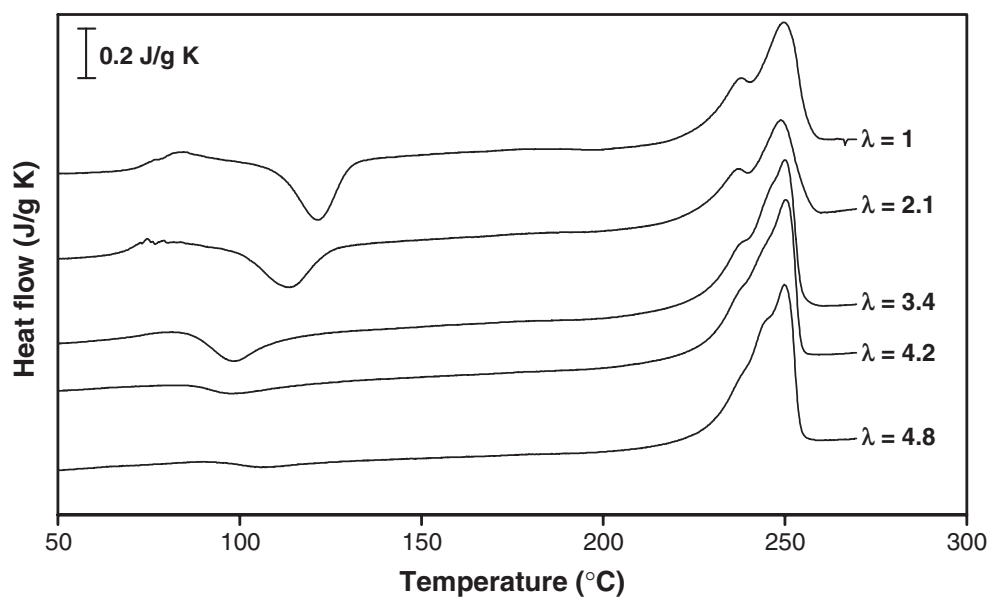

Figure 5. DSC normalized curves for PET-MXD6-ion, $\mathrm{Zn}^{2+}$ blends: $88 / 10 / 2$ drawn at various draw ratios.

The MXD6 DSC curve is a function of $\lambda$ and is similar to that observed for PET. Increasing the draw ratio shifts the cold crystallization temperature toward the $T_{\mathrm{g}}$ with a concomitant decreasing of the crystallization enthalpy. Beyond $\lambda=4.6$, the cold crystallization peak disappears. The melting temperature remains constant for each draw ratio. The shape of the peak of fusion is also different for the different materials: for $\lambda<3.3$ samples, the peak is asymmetric, while for the $\lambda>3.3$ samples it appears as due to the superimposition of two fusion reactions. This phenomenon is also sometimes observed in drawn PET $[10,13]$. Indeed, two peaks of fusion have been observed for wet-drawn PET while only one peak has been observed for dry-drawn PET. We can assume that, as in PET, the presence of water molecules has impeded the crystal growth during drawing and led to the appearance of crystallites of smaller sizes (as MXD6 is highly hydrophilic and some water content is probably present during the drawing). These crystallites have a lower temperature of fusion.

On the DSC curves of drawn blends shown in Figure 5, it should be also noticed that while the crystallization exotherm shifts to lower temperatures with increasing draw, the peak area decreases with drawing and the cold crystallization peak becomes very weak above $\lambda=4.2$.

Figures 6-8 gather, respectively, the variations of cold crystallization enthalpy $\left(\Delta H_{\mathrm{c}}\right)$, temperature at the minimum of the cold crystallization peak $\left(T_{\mathrm{c}}\right)$, and of the melting enthalpy $\left(\Delta H_{\mathrm{m}}\right)$ with the draw ratio for the 


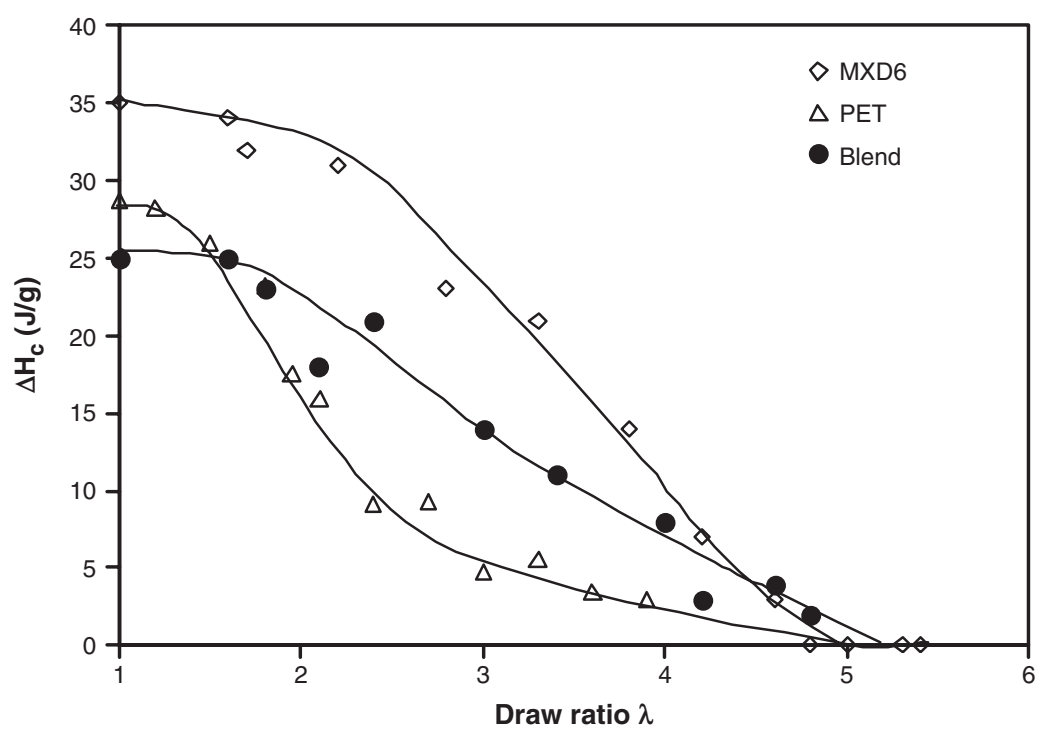

Figure 6. Enthalpy of the cold crystallization peaks vs draw ratio for PET, MXD6, and PET-MXD6-ion, $\mathrm{Zn}^{2+}$ blends (88/10/2).

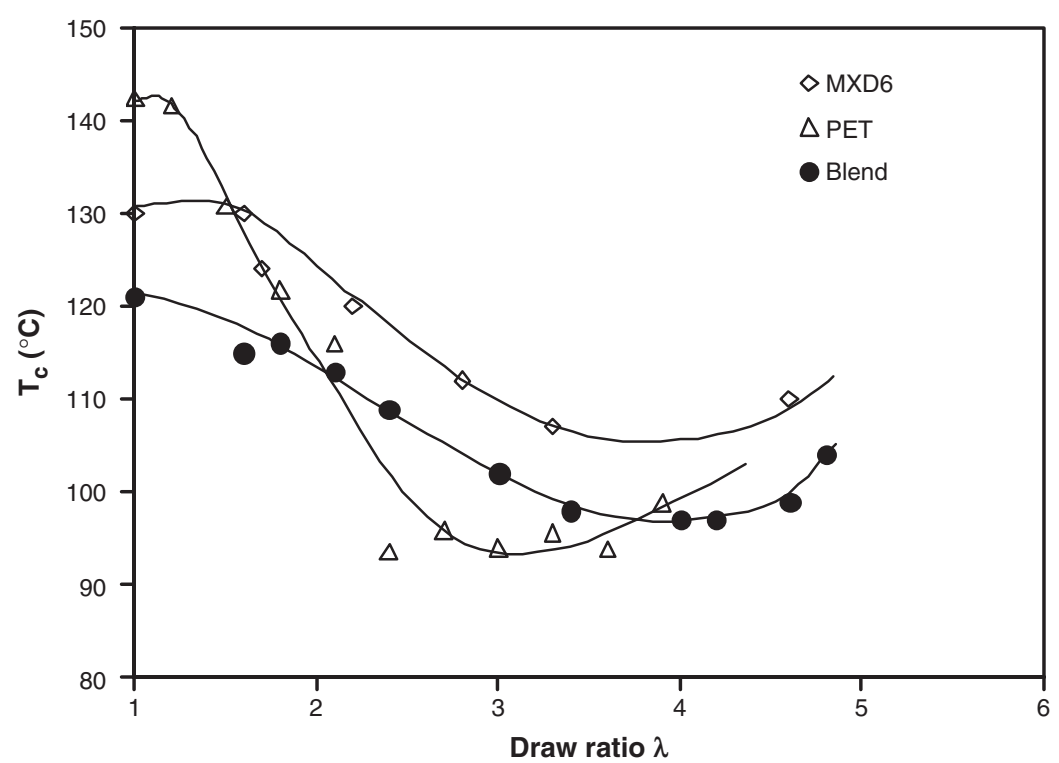

Figure 7. Temperature $T_{\mathrm{c}}$ vs draw ratio for PET, MXD6, and PET-MXD6-ion, $\mathrm{Zn}^{2+}$ blends $(88 / 10 / 2)$. 


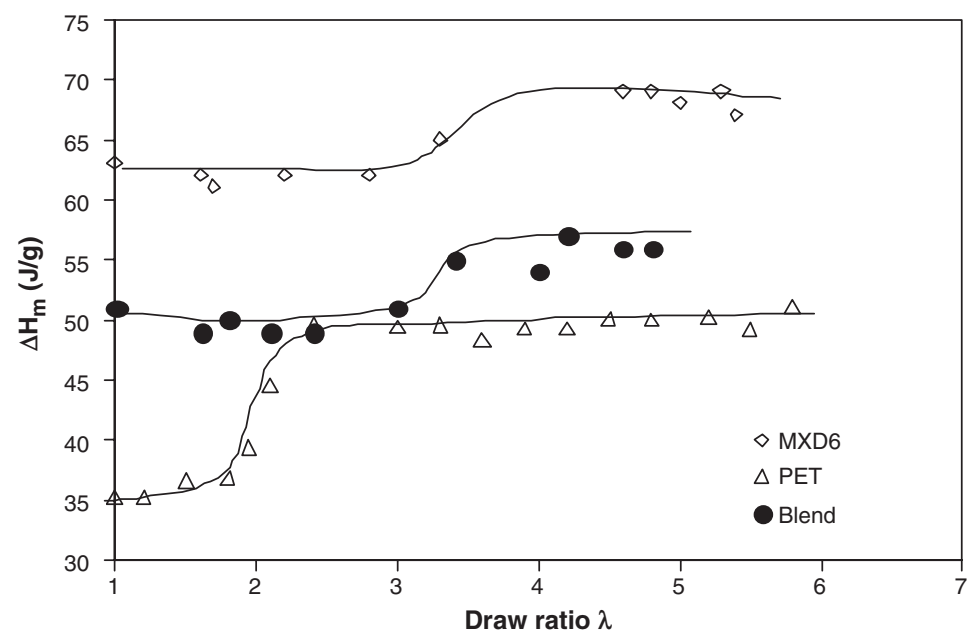

Figure 8. Melting enthalpy vs draw ratio for PET, MXD6, and PET-MXD6-ion, $\mathrm{Zn}^{2+}$ blends $(88 / 10 / 2)$.

Table 3. Critical values of the three materials determined by DSC and birefringence measurements. Value of $\lambda$ for the lower $T_{c}$ value.

\begin{tabular}{lccc}
\hline & PET & MXD6 & Blend \\
\hline Birefringence & & & \\
$\lambda_{\mathrm{c} 1}$ & 2.3 & 3 & $-^{*}$ \\
$\lambda_{\mathrm{c} 2}$ & 4.3 & 4.7 & $-^{*}$ \\
$\mathrm{DSC}\left(X_{\mathrm{c}}\right)$ & & & 2.5 \\
$\lambda_{\mathrm{c} 1}^{\prime}$ & 2 & 2.3 & 4.4 \\
$\lambda_{\mathrm{c} 2}^{\prime}$ & 4.2 & 4.6 & $3.8<\lambda<4.4$ \\
Value of $\lambda$ for the lower $T_{\mathrm{c}}$ value & $2.5<\lambda<3.3$ & $3.3<\lambda<4.2$ & $3.3<$ \\
\hline
\end{tabular}

*For the blend, the birefringence could not be determined due to haze of the samples.

three materials. Whatever be the sample, $\Delta H_{\mathrm{c}}$ decreases with increasing $\lambda$ (Figure 6). For PET, the cold crystallization is negligible above $\lambda=3$ [13], while for MXD6 and the blend, $\Delta H_{\mathrm{c}}$ is negligible for $\lambda>4.5$.

The plots of cold crystallization temperature changes with $\lambda$ show two domains (Figure 7). In the first domain, the cold crystallization peak decreases to a lower temperature as $\lambda$ increases, while in the second domain, the peak increases. For PET, when $\lambda$ increases from 1 to 3 , $T_{\mathrm{c}}$ decreases to $95.5^{\circ} \mathrm{C}$ and then increases with $\lambda$ until $T_{\mathrm{c}}=99^{\circ} \mathrm{C}$. For MXD6, $T_{\mathrm{c}}$ decreases for $1<\lambda<4$ until it reaches $107^{\circ} \mathrm{C}$; it then increases with $\lambda$ until $T_{\mathrm{c}}=110$. For the blend, $T_{\mathrm{c}}$ decreases for $1<\lambda<4$ until it reaches $97^{\circ} \mathrm{C}$; it then increases with $\lambda$ until $T_{\mathrm{c}}=104^{\circ} \mathrm{C}$ (see Table 3). 
Thermal cold crystallization is known to be sensitive to the existence of a pre-orientation of the molecular chains, which is a factor contributing to the decrease of the thermal crystallization temperature in the first domain [13]. In the second domain, the SIC takes places during the drawing, which reduces the ability to crystallize during the DSC heating. Thus, the enthalpy of the extra-cold crystallization is reduced and its temperature slightly increased [13].

From the data reported in Figure 8, we observe that the melting enthalpy varies with $\lambda$. For a low draw ratio $(\lambda<2$ for PET; $\lambda<3.3$ for MXD6 and blend), $\Delta H_{\mathrm{m}}$ is constant and equal to the melting enthalpy of an undrawn material, while at a high draw ratio $(\lambda>4.5$ for MXD6 and the blend, $\lambda>2.5$ for PET), $\Delta H_{\mathrm{m}}$ reaches the maximum values.

To examine orientation development during drawing of MXD6 and PET, birefringence has been measured for drawn MXD6 and PET. Indeed, birefringence measurement is the important method to evaluate both the orientation and the relaxation phenomena of polymers [22]. Birefringence is due to a difference between the principal refractive indices within a material and its variations can be interpreted in this work in regard to average orientation of the macromolecules. For uniaxial sample deformation, the birefringence due to orientation, $\Delta n$, is defined as:

$$
\Delta_{n}=n_{\|}-n_{\perp}
$$

where $n_{\|}$and $n_{\perp}$ are, respectively, the refractive indices parallel and perpendicular to the stretching direction. Birefringence data obtained for PET and MXD6 samples are reported in Figure 9. It should be noticed that PET exhibits three regimes during the drawing. First, for $\lambda$ values up to $\approx 2.3, \Delta n$ increases from $\Delta n=0$ to $\Delta n \approx 0.05$. For the critical value $\lambda_{\mathrm{c} 1} \approx 2.3$, a slope change occurs, and the birefringence drastically changes. The birefringence increases up to $\Delta n \approx 0.225$. During regime II and between $\lambda=3$ and $\lambda=4$, a significant decrease of the slope is observed and discussed here. For $\lambda=4.3$, a second distinctive discontinuity occurs: this is the second critical value called $\lambda_{\mathrm{c} 2}$. After this value, $\Delta n$ will remain constant. Similar variations are observed for MXD6. We notice a weak shift of $\lambda_{\mathrm{c} 1}$ and $\lambda_{\mathrm{c} 2}$ toward higher draw ratio values leading to $\lambda_{\mathrm{c} 1} \approx 3$ and $\lambda_{\mathrm{c} 2} \approx 4.7$ for the first and second steps, respectively. The maximum birefringence is clearly weaker than those observed for PET ( $\Delta n \approx 0.08$ ). Finally, for a given draw ratio, the values of $\Delta n$ observed for a PET sample are always higher than those obtained for the MXD6 sample. 


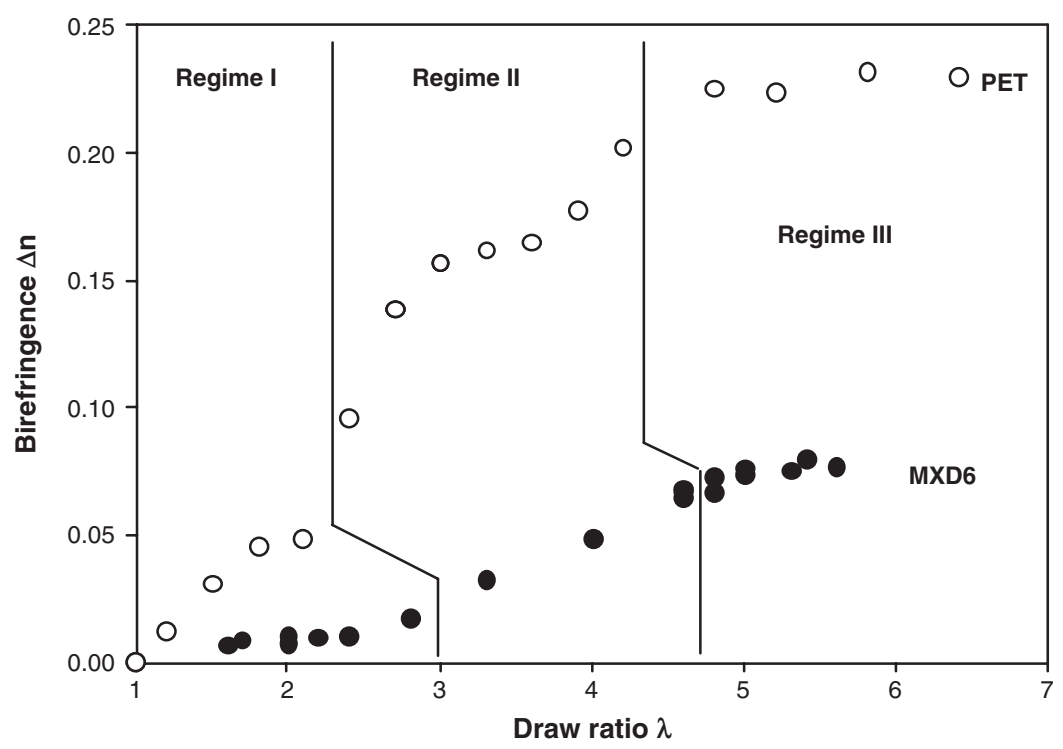

Figure 9. Birefringence vs draw ratio for PET and MXD6 films drawn at $100^{\circ} \mathrm{C}$.

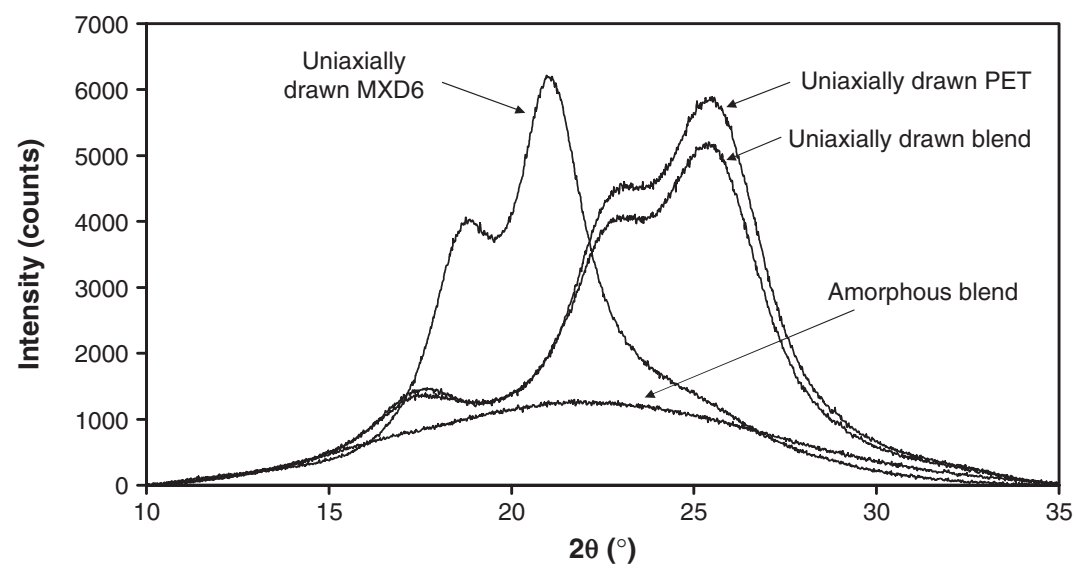

Figure 10. XRD curves for amorphous blend, blend crystallized by drawing $(\lambda=5.2)$, and drawn PET and MXD6 films.

The XRD curves $(I=f(2 \theta))$ of an undrawn PET-MXD6 blend shows (in Figure 10) a shape, characteristic of an amorphous polymer, which consists in a hump observable between $2 \theta=10^{\circ}$ and $2 \theta=35^{\circ}$. For highly drawn PET and MXD6, peaks characteristics of their crystalline phases 
are observed. A drawn blend $(\lambda=5.2)$ shows the existence of three peaks superimposed on the amorphous hump (at $2 \theta=17.4^{\circ}, 23^{\circ}$, and $25.4^{\circ}$ ). These peaks are similar to the peak obtained for PET, which composed $88 \%$ of the blend. The presence of the ionomer and the MXD6 nodules does not seem to have modified the SIC of PET. No evidence for a SIC of the MXD6 nodules is given here. It could be due to the fact that the MXD6 nodules remain amorphous or to the weak percentage in mass (10\% of MXD6 in the blend) of the MXD6 crystalline phase.

X-ray diffraction analysis carried out on undrawn PET and MXD6 show a curve similar to the shape observed in Figure 10. The X-ray patterns do not exhibit any peak that could be attributed to the existence of the crystalline phase. So the undrawn materials are initially amorphous. The birefringence measurements taken on the injection molded plates, $\Delta n=0$, proves that the macromolecular chains of the PET and the MXD6 are disordered and randomized. So the initial plates are considered as globally isotropic. For the undrawn blend, XRD analysis shows a shape characteristic of an amorphous polymer. The presence of an exothermic peak in DSC curve confirms the assumption that this undrawn blend is amorphous and crystallizable during heating. For MXD6, the disappearance of the cold crystallization peak when $\lambda$ increases and the presence of the fusion peak probably indicates the presence of a SIC phase. This has already been observed in previous works [9-15]. A similar evolution can be postulated for MXD6. This is confirmed by the shape of the XRD curve for a $\lambda=5.2$ blend sample, where the response of the SIC is observed (Figure 10). From these DSC results, we can assume that the SIC appears equal to between 2.5 and 3.5 for both MXD6 and blend (see Table 3).

The birefringence measurements show how the orientation develops during drawing of PET and MXD6. According to Cakmak [23], for the first regime $\left(\lambda<\lambda_{\mathrm{c} 1}\right)$ observed in Figure 8 , the material is considered as isotropic and amorphous. However, in this drawing zone the birefringence increases linearly with $\lambda$, revealing a gradual growth of the macromolecular orientation with the polymeric chain axis becoming progressively more parallel to the draw direction. In the first part of the second regime $\left(\lambda_{\mathrm{c} 1}<\lambda<\lambda_{\mathrm{c} 2}\right)$, the rapid increase of birefringence was already and clearly attributed to the emergence of a SIC phase, which grows to the detriment of the amorphous phase when $\lambda$ increases. In a previous work [14], we have clearly shown by pole figure and DSC analysis that after the appearance of the crystallites, there is an alignment of the crystalline structure with the draw direction without important modification of the degree of crystallinity. This explains the variations of the slope in this regime. The third regime $\left(\lambda>\lambda_{\mathrm{c} 2}\right)$ 
is reached when finite extensibility brings about saturation of birefringence while stress continues to increase. For the blend, Figure 4 shows that as the drawing increased, the crystallization exotherm shifted to lower temperatures and the peak area decreased. Since the same behavior was observed for the blend as for the two components of the blend, this decrease can be attributed to an increase of the degree of crystallinity. We have calculated the degree of crystallinity for these samples with Equation (2).

$$
X_{\mathrm{c}}=\frac{\Delta H_{\mathrm{c}}^{0}-\Delta H_{\mathrm{c}}}{\Delta H_{\mathrm{c}}^{0}} \cdot \frac{\Delta H_{\mathrm{m}}}{\Delta H_{\mathrm{m}}^{0}}
$$

where

$\Delta H_{\mathrm{m}}=$ melting enthalpy of a drawn sample

$\Delta H_{\mathrm{c}}=$ enthalpy of cold crystallization

$\Delta H_{\mathrm{c}}^{0}=$ enthalpy of cold crystallization of an amorphous and isotropic sample

$\Delta H_{\mathrm{m}}^{0}=$ heat of fusion for a $100 \%$ crystalline polymer.

The values of calculated melting enthalpy of a wholly crystalline PET and crystallization enthalpy of the wholly amorphous PET are 140 and $29 \mathrm{~J} / \mathrm{g}$, respectively [24] whereas there is no value of $\Delta H_{\mathrm{m}}^{0}$ for MXD6. Nevertheless, we have shown in a previous work that the $\Delta H_{\mathrm{m}}^{0}$ can be obtained from an extrapolation method [25]. Thus, from the XRD and DSC values of various semicrystalline samples, $\Delta H_{\mathrm{m}}^{0}$ for MXD6 is determined and is equal to $175 \mathrm{~J} / \mathrm{g}$.

Using the values of $\Delta H_{\mathrm{c}}$ and $\Delta H_{\mathrm{m}}$ determined in this work (Figures 6 and 8), we can estimate the degree of crystallinity $\left(X_{\mathrm{c}}\right)$ reached after each drawing period for PET, MXD6, and the blend. Figure 11 shows three domains for the different series of samples, defining two critical values of the draw ratio: (1) $\lambda_{c 1}^{\prime}$ critical draw ratio for which a SIC appears and (2) $\lambda_{\mathrm{c} 2}^{\prime}$ critical draw ratio for which the degree of crystallinity reached by the sample is maximum. For $X_{\mathrm{c}}$ up to $\lambda=\lambda_{\mathrm{c} 1}^{\prime}$, the materials are still regarded as amorphous; then in the second phase $X_{\mathrm{c}}$ increases to reach $40 \%$ for $\lambda_{\mathrm{c} 2}^{\prime}$. Finally, for $\lambda>\lambda_{\mathrm{c} 2}^{\prime}, X_{\mathrm{c}}$ is constant (the cold crystallization enthalpy vanishes, and the melting enthalpy reaches its maximum). The values of $\lambda_{\mathrm{c} 1}^{\prime}$ and $\lambda_{\mathrm{c} 2}^{\prime}$, presented in Table 3 for the different materials, are very close to the critical values of $\lambda_{1}$ and $\lambda_{2}$ of draw ratio $\lambda$ determined by birefringence measurements.

For these immiscible blends, XRD patterns show only the SIC of the PET fraction. The scanning electron micrograph shown in Figure 12 shows the microstructure of a $\lambda=4.4$ drawn blend. It is noted that the 


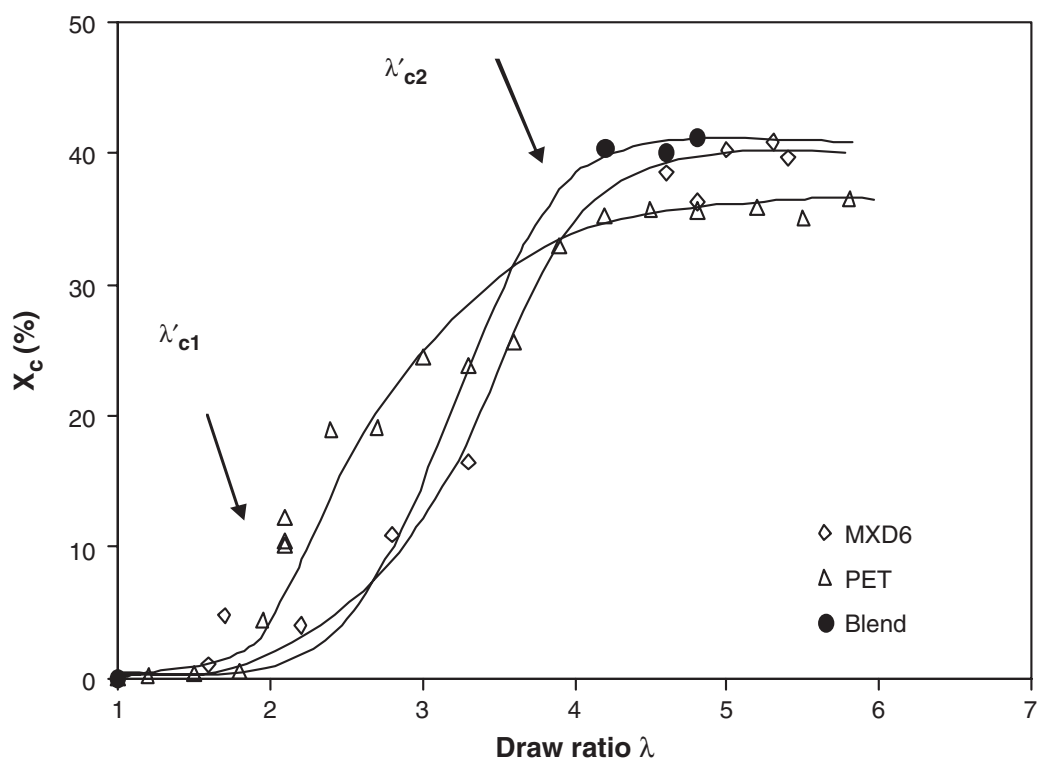

Figure 11. DSC degree of crystallinity vs draw ratio.

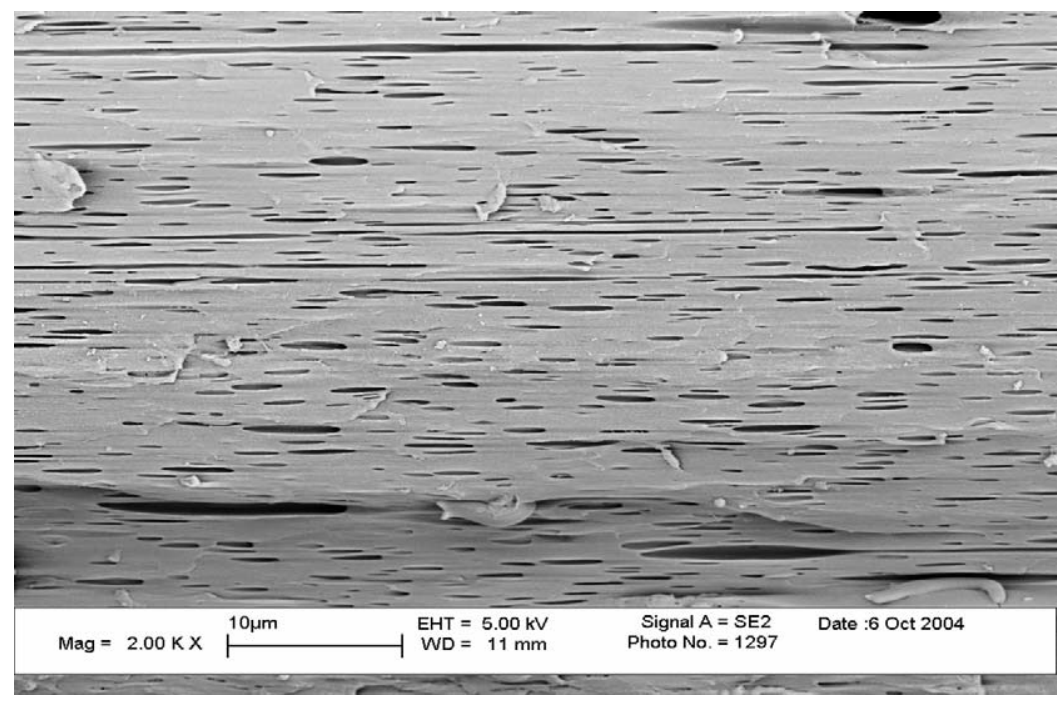

Figure 12. Scanning electron micrograph of uniaxially hot drawn PET-MXD6-ionomer blends $(88 / 10 / 2)(\lambda=4.4)$. 
spherical nodules of MXD6 are transformed into lamellar tiles oriented in the direction of drawing. The average size of tiles is $\approx 4 \mu \mathrm{m}$ in length and $0.3 \mu \mathrm{m}$ in width. A draw ratio of the nodules is calculated on numerous nodules and is $\lambda=4.5$. So different results speak for a SIC of the MXD6 fraction:

- the nodules are drawn and the draw ratio of the nodules is the same as the macroscopic draw ratio

- the values of $\lambda_{\text {c1 }}^{\prime}$ are close for the two polymers, which indicated a similar behavior during drawing.

The fractional crystallinity of drawn blend samples is theoretically equal to the sum of both fractional crystallinities of PET and MXD6:

$$
X_{\mathrm{c}_{\text {blend }}}=X_{\mathrm{c}_{\mathrm{PET}}}+X_{\mathrm{c}_{\mathrm{MXD} 6}}
$$

It is necessary to determine the degree of crystallinity of each component. This is possible for the highly drawn sample $\left(\lambda>\lambda_{\mathrm{c} 2}^{\prime}\right)$ by a separation of the melting peak of the blend into two peaks corresponding to the fusion of the each crystalline phase. For the low draw ratio $\left(\lambda<\lambda_{\mathrm{c} 2}^{\prime}\right)$ a complex cold crystallization peak is observed. This peak cannot be easily separated into two components. For the calculation of $X_{\mathrm{c}}$ of the blend, therefore, we took only the high stretched samples $\left(\lambda>\lambda_{\mathrm{c} 2}^{\prime}\right.$ and $\left.\Delta H_{\mathrm{c}}=0 \mathrm{~J} / \mathrm{g}\right)$ :

$$
X_{\mathrm{c}_{i}}=\frac{\Delta H_{\mathrm{m}_{i}}}{\Delta H_{\mathrm{m}_{i}}^{0}}
$$

One observes similar variations to those of each component and the maximum degree of crystallinity for the blend is similar to that of pure polymers and it is close to $41 \%$. This shows that the SIC of this drawn blend occurs in each PET and MXD6 domains as in the two pure materials. For the whole highly drawn blend, it gives $4 \%$ in mass for the crystalline phase of the MXD6 fraction. This weak percentage is probably the reason for which no XRD response is observed. A 70/30 PET-MXD6 blend must be studied to verify this conclusion.

The appearance of the SIC phase leads to vanish the thermal crystallization of the amorphous phase. Nevertheless, Figures 5 and 6 show that a cold crystallization remains possible. Although the SIC has not begun for $\lambda<\lambda_{\mathrm{c} 1}^{\prime}$, this extra crystallization takes place through a spherulitic way. For $\lambda>\lambda_{\mathrm{c} 1}^{\prime}$, this crystallization occurs by growth of the SIC phase $[12,14,15]$. 
Concerning amorphous and undrawn samples, the cold crystallization temperature of the blend is lower (by $17^{\circ} \mathrm{C}$ ) than the average $T_{\mathrm{c}}$ of the two components. This may be due to two reasons:

- the presence of the compatibilizer

- a geometrical effect due to an increase of interfaces due to the inclusions of MXD6 in PET matrix, which enhances the crystallization rate.

On drawn materials, the variation of the thermal crystallization temperature is sensitive to the existence of a preorientation of the molecular chains, which is a factor contributing to the decrease of the thermal crystallization temperature [24]. In the strain-induced crystallized samples, the presence of the crystallites leads to a weak increase of the temperature of thermal crystallization $\left(T_{\mathrm{c}}\right)$ with $\lambda_{\mathrm{c} 1}^{\prime}<\lambda<\lambda_{\mathrm{c} 2}^{\prime}$.

\section{CONCLUSIONS}

In this study, the microstructure of uniaxially hot-drawn PET-MXD6 films was characterized by DSC, birefringence measurements, and $\mathrm{X}$-ray diffraction. As in PET, an uniaxial hot drawing of the MXD6 and the PET-MXD6 blends leads to the appearance of a strain-induced crystalline phase for a given draw ratio. This crystallization takes place between $\lambda_{\mathrm{c} 1}$ and $\lambda_{\mathrm{c} 2}$, the second being the critical value of the draw ratios for which the maximum crystallization is fully achieved.

The analysis of the crystallization by DSC shows a similar thermal behavior between PET, MXD6 and the blends, which leads to the same critical value. Moreover, it has been shown that PET and MXD6 crystallize separately in the blends. The maximum degree of crystallinity reached by drawing is close to $40 \%$ for the three materials.

\section{ACKNOWLEDGMENT}

The authors would like to thank Eric Grollier (Laboratoire Etudes et Essais, Tergal Fibers, France), who provided the pellets of PET (grade T74F9) and 'Réseau Matériaux Polymères Plasturgie' for its financial support. 


\section{REFERENCES}

1. Patcheak, T.D. and Jabarin, S.A. (2001). Structure and Morphology of PET/PEN Blends, Polymer, 42(21): 8975-8985.

2. Mitsubishi Gas Chemical Co., Inc. N-MXD6 as a Barrier Material for Films, N-MXD6 Technical Information for Film Application, MF-01E.

3. Mitsubishi Gas Chemical Co., Inc. N-MXD6 as a Barrier Material for PET Bottles, Technical Information for PET Bottle Applications, MB-01E.

4. Samios, C.K. and Kalfoglou, N.K. (1999). Compatibilization of Poly(ethylene terephtalate)/Polyamide-6 Alloys: Mechanical, Thermal and Morphological Characterization, Polymer, 40(17): 4811-4819.

5. Huang, C.C. and Chang, F.C. (1997). Reactive Compatibilization of Polymer Blends of Poly(butylene terephtalate) and Polyamide 6,6:2. Morphological and Mechanical Properties, Polymer, 38(17): 4287-4293.

6. Evstatiev, M., Schultz, J.M., Petrovich, S., Georgiev, G., Fakirov, S. and Friedrich, K. (1998). In situ Polymer/Polymer Composites from Poly (ethylene terephtalate), Polyamide-6, and Polyamide-66 Blends, J. Appl. Polym. Sci., 67(4): 723-737.

7. Liu, R.Y.F., Hiltner, A. and Baer, E. (2004). Free Volume and Oxygen Transport in Cold-drawn Polyesters, J. Polym. Sci. Part B: Polym. Phys., 42(3): 493-504.

8. Kawakami, D., Hsiao, B.S., Ran, S., Burger, C., Fu, B., Sics, I., Chu, B. and Kikutani, T. (2004). Structural Formation of Amorphous Poly(ethylene terephtalate) during Uniaxial Deformation above Glass Temperature, Polymer, 45(3): 905-918.

9. Lu, X.F. and Hay, J.N. (2001). Crystallization Orientation and Relaxation in Uniaxially Drawn Poly(ethylene terephtalate), Polymer, 42(19): 8055-8067.

10. Zumailan, A., Dargent, E. and Saiter, J.M. (2004). Characterization of Polyethylene Terephthalate Films Drawn in Hot Water, Polym. Eng. Sci., 44(2): 223-230.

11. Dargent, E., Grenet, J. and Auvray, X. (1994). Thermal Behaviour of Drawn Semi-crystalline Poly(ethylene terephthalate) Films, J. Therm. Anal., 41: 1409-1415.

12. Dargent, E., Denis, A., Galland, C. and Grenet, J. (1996). Non-isothermal Crystallization Kinetics of Hot Drawn Polyester Films: Kissinger and Ozawa Analysis, J. Therm. Anal., 46: 377-385.

13. Dargent, E., Denis, G., Caron, C., Saiter, J.M. and Grenet, J. (2000). Effect of Water Molecules on Crystallization during Uniaxial Drawing of Poly(ethylene terephthalate) Films, J. Appl. Polym. Sci., 77(5): 1056-1066.

14. Dargent, E., Grenet, J. and Dahoun, A. (1997). Evolution of Hot Strain Induced Crystalline Texture of Poly(ethylene terephthalate) Films, Polym. Eng. Sci., 37(11): 1853-1857. 
15. Kattan, M., Dargent, E. and Grenet, J. (2002). Three Phase Model in Drawn Thermoplastic Polyesters: Comparaison of Differential Scanning Calorimetry and Thermally Stimulated Depolarisation Current Experiments, Polymer, 43(4): 1399-1405.

16. Subramanian, P.M. (1987). Poly(ethylene terephthalate) Blends for Permeability Barrier Applications, Polym. Eng. Sci., 27(21): 1574-1581.

17. Kalfoglou, N.K. and Skafidas, D.S. (1994). Compatibility of Blends of Poly(ethylene terephthalate) with the Ionomer of Ethylene-Methacrylic Acid Copolymer, Eur. Polym. J., 30(8): 933-939.

18. Wilis, J.M. and Favis, B.D. (1988). Processing-morphology Relationships of Compatibilized Polyolefin/Polyamide Blends. Part I: The Effect of an Ionomer Compatibilizer on Blend Morphology, Polym. Eng. Sci., 28(21): 1416-1426.

19. Molnar, A. and Eisenberg, A. (1992). Miscibility of Polyamide-6 with Lithium or Sodium Sulfonated Polystyrene Ionomers, Macromolecules, 25(21): 5774-5782.

20. Hay, I.L. (1980). In: Fava, R.A. (ed.), Methods of Experimental Physics, Vol. 16, Part C, p. 163, Academic Press, New York.

21. Persyn, O., Miri, V., Lefebvre, J.M., Depecker, C., Gors, C. and Stroeks, A. (2004). Structural Organization and Drawability in Polyamide Blends, Polym. Eng. Sci., 44(2): 261-271.

22. Oultache, A.K., Kong, X., Pellerin, C., Brisson, J., Pézolet, M. and Prud'homme, R.E. (2001). Orientation and Relaxation of Orientation of Amorphous Poly(ethylene terephthalate), Polymer, 42(21): 9051-9058.

23. Cakmak, M. (2004). Polymer Films and Fibers Symposium, Montréal, Canada, Sept. 25-27.

24. Wunderlich, B. (1980). Macromolecular Physics, Vol. 3, Academic Press, New York.

25. Ben Doudou, B., Dargent, E., Marais, S., Grenet, J. and Hirata Y. Barrier Properties and Microstructure Modifications Induced by Liquid Water for a Semi-aromatic Polyamide, J. Polym. Sci. Part B: Polym. Phys. (in press).

\section{BIOGRAPHIES}

\section{Bessem Ben Doudou}

Bessem Ben Doudou is in his third year as a PhD candidate at the University of Rouen. He is studying the physical properties of PET and polyamide blends to obtain drawn materials with high gas barrier properties. 


\section{Eric Dargent}

Eric Dargent received his $\mathrm{PhD}$ in Polymer Physics from the University of Rouen in 1994. He works in the field of polymer microstructure and the glass transition of semicrystalline thermoplastic polymers. Eric is a Research Officer in the LECAP team of the Material Institute of Rouen, CNRS 6522 'Polymers, Biopolymers and Membranes'.

\section{Jean Grenet}

Professor Jean Grenet holds his PhD from the University of Rouen in Physics. He was the leader of the LECAP team. He has authored over 80 refereed papers in the area of thermal analysis, glass forming liquid properties, and mechanical properties of polymers. Jean is currently a Research Officer of the Material Institute of Rouen, CNRS 6522 'Polymers, Biopolymers and Membranes' http://www.univ-rouen.fr/ pbm/. 